\title{
Effects of Annealing on Electrical and Optical Properties of a Multilayer InAs/GaAs Quantum Dots System
}

\author{
Adenilson José Chiquito ${ }^{\mathrm{a} *}$, Yuri Alexander Pusep ${ }^{\mathrm{b}}$, Sérgio Mergulhão ${ }^{\mathrm{a}}$, \\ Yara Galvão Gobato ${ }^{a}$,José Cláudio Galzerania , Nicolai Moshegov ${ }^{\mathrm{c}}$ \\ ${ }^{a}$ Departamento de Física, Universidade Federal de São Carlos, \\ CP 676, 13565-905 São Carlos - SP, Brazil \\ ${ }^{\mathrm{b}}$ Instituto de Física de São Carlos, Universidade de São Paulo, \\ 13650-970 São Carlos - SP, Brazil \\ ${ }^{\mathrm{c}}$ The Pennsylvania State University, Pennsylvania,16802
}

Received: December 8, 2003; Revised: March 21, 2004

\begin{abstract}
A systematic investigation of the properties of the InAs/GaAs self-assembled quantum dots (SAQDs) system subjected to a post-growth annealing using capacitance-voltage, Raman scattering and photoluminescence measurements is presented. The application of both electrical and optical methods allowed us to obtain reliable information on the microscopic structural evolution of this system. The single layer and the multilayer quantum dots were found to respond differently to the annealing process, due to the differences in strain that occur in both systems. The diffusion activated by strain provoked the appearance of an InGaAs alloy layer in substitution to the quantum dots layers; this change occurred at the annealing temperature $\mathrm{T}=600{ }^{\circ} \mathrm{C}$ in the multilayer system. A single dot layer, however, was observed even after the annealing at $\mathrm{T}=700^{\circ} \mathrm{C}$. Moreover, the low temperature annealing was found to improve the homogeneity of the multilayer system and to decrease the electrical interlayer coupling.
\end{abstract}

Keywords: InAs/GaAs quantum dots, capacitance, raman scattering

\section{Introduction}

In recent years, self-assembled quantum dots (SAQDs) have attracted much attention due to their peculiar electronic and optical properties interesting both to fundamental physics and technological applications. The formation of the dots is usually based on the transformation of a strained layer into an assembly of islands of atoms when the two dimensional growth changes into a three dimensional one. This is the so-called Stranski-Krastanow growth mode'.

The strong quantum confinement of the carriers presented by these defect-free quantum dots makes them an important structure for the development of electronic devices. However, the fabrication of reproducible homogeneous SAQDs structures suitable for applications remains to be achieved. Usually, in order to control the homogeneity of the dots, both the deposition rate and the substrate temperature are varied during the growth process. On the other hand, the structure of the SAQDs can be made uniform by a post-growth annealing. As a matter of fact, the influence of the annealing was reported using the combination of photoluminescence (PL) and transmission electron microscopy ${ }^{2-6}$. Homogeneous samples were shown to occur due to the atomic interdiffusion process between the barriers and the islands (dots) which changes the sizes and the shapes of the dots. Thus, the controlled heat treatment presents a way to achieve a quantum dot device with tailored features, since a controlled annealing process can tune the electronic properties. However, it is not yet clear how important are the relative modifications in the barriers (host crystal) and in the SAQDs itself, attained after the annealing.

The characteristics of multilayer InAs/GaAs SAQDs structures annealed after the growth were here studied using a combination of capacitance-voltage $(\mathrm{C}-\mathrm{V})$ measurements, Raman scattering and PL spectroscopy. Both the C-V measurements and the PL spectroscopy are sensitive to the electronic properties of the studied systems; however the former, in addition, provides useful information about their

*e-mail: chiquito@df.ufscar.br 
local electrical behavior. Meanwhile, the phonon Raman scattering is a widely recognized structural sensitive tool. As we shall see, a relatively low annealing temperature $\left(500^{\circ} \mathrm{C}\right)$ improved the homogeneity of the electron distribution and caused the decrease of the electrical coupling between the planes containing the dots. The Raman scattering, sensitive to structural modifications, provided evidences of a reorganization of the SAQDs system (SAQDs + barriers). However, in contrast to the data presented in Refs. 3,4 where the dots were found to survive even after the annealing at rather high temperatures $\left(700-900{ }^{\circ} \mathrm{C}\right.$ ) in multilayer SAQDs structures, we observed their disappearance already at $600{ }^{\circ} \mathrm{C}$. A much higher temperature resistance to the annealing temperature was indeed observed in a single layer structure. We suppose that the thermal stability of the SAQDs structure strongly depends on the growth conditions resulting in different strain distributions over the InAs/GaAs structure.

\section{Experiment}

The samples were grown by a MECA 2000 molecularbeam epitaxy system on (100) GaAs highly doped substrates; they were based on planes containing the InAs SAQDs embedded in a field effect device. The five InAs layers with nominal thicknesses 2.3 monolayers (ML) separated by $15 \mathrm{ML}$ 's of GaAs were grown at $450{ }^{\circ} \mathrm{C}$. The process of the dots formation was monitored by reflection of high-energy electron diffraction (RHEED): the transition from a streaked to a spotty RHEED pattern, indicating the formation of 3D islands, was observed after the deposition of 1.8 ML thick InAs. After the growth of SAQDs, an undoped GaAs separating layer $(25 \mathrm{~nm})$ was grown, followed by an undoped GaAs/AlAs $(1 \mathrm{~nm} / 3 \mathrm{~nm})$ superlattice in order to increase the electrical impedance of the samples. Finally, the structure was capped with a $5 \mathrm{~nm}$ GaAs layer in order to prevent the surface oxidation. During the growth, the InAs and GaAs fluxes were fixed at 0.1 and $0.35 \mathrm{ML} / \mathrm{s}$ respectively, while $\mathrm{P}_{\mathrm{As}}=5 \times 10^{-6} \mathrm{~Pa}$. The used samples (labeled A and B) are distinguished by different $\mathrm{Si}$ doping levels of the GaAs regions where the dots were grown: $N_{D}^{A}=5 \times 10^{17} \mathrm{~cm}^{-3}$ and $N_{D}^{B}=1 \times 10^{17} \mathrm{~cm}^{-3}$.

The post-growth annealing process was done in an argon atmosphere at different temperatures during $20 \mathrm{~min}$. A control sample with only a single InAs plane was grown in a similar way to those described above.

For the Schottky diode construction, we fabricated a conventional ohmic contact to the substrate with an AuGeNi alloy annealed at $450{ }^{\circ} \mathrm{C}$ for $120 \mathrm{~s}$; the Schottky contact was formed by the deposition of a $200 \mathrm{~nm}$ gold layer with $500 \mu \mathrm{m}$ diameter. The capacitance was measured using standard lock-in techniques (with a SR530 Stanford dualphase lock-in amplifier). The samples were mounted in a variable temperature cryostat (Janis CCS350) and the C-V curves were obtained at $10 \mathrm{~K}$.

Unpolarized back-scattering Raman spectra were performed at $10 \mathrm{~K}$ with a Jobin Yvon T64000 triple spectrometer supplied with a charge coupled device (CCD) detector cooled by liquid nitrogen; PL measurements were also performed at $10 \mathrm{~K}$ with a Spex 500M single spectrometer using a photomultiplier tube. The $514.5 \mathrm{~nm}$ and $457.9 \mathrm{~nm}$ lines of an $\mathrm{Ar}^{+}$laser were used for excitation (Raman and PL, respectively). In order to measure Raman and PL spectra, the top layers containing the GaAs/AlAs superlattice were first removed.

\section{Results and Discussion}

In order to deal with the $\mathrm{C}-\mathrm{V}$ measurements, the spatial distribution of the electrons along the growth direction was calculated by the usual equations for the $\mathrm{C}-\mathrm{V}$ profile ${ }^{7}$,

$$
N_{C-V}(z)=\frac{-2}{q S^{2} \varepsilon_{S}}\left[\frac{d C^{-2}}{d V}\right]^{-1}
$$

and

$$
z=\frac{\varepsilon_{S} S}{C}
$$

where $C$ is the measured capacitance, $S$ is the Schottky device area and the other symbols have their usual meanings.

Figure 1 shows the $\mathrm{C}-\mathrm{V}$ profiles of samples $\mathrm{A}$ and $\mathrm{B}$ : it is possible to distinguish individual contributions of the InAs planes containing the dots located at $164 \mathrm{~nm}, 170 \mathrm{~nm}$ and $175 \mathrm{~nm}$ from the surface (Fig. 1a). The positions of the peaks were found in accordance with the nominal localization of the second, third, and fourth InAs planes (indicated by the arrows). The first InAs layer in both samples does not contribute to the $\mathrm{C}-\mathrm{V}$ profile because the built-in field produced by the Schottky contact has already depleted it. The profile width was used in order to evaluate an effective length $(\Delta z)$ which can serve as an estimate of the dots height: we observed a mean height $\sim 4 \mathrm{~nm}$ (see for instance Ref. 8). The C-V profile of sample B is depicted in Fig. 1b. In this case, as a consequence of the lower electron concentration, the screening effects did not allow us to resolve individual contributions of the SAQDs layers as in sample A. Figure 1 also presents the $\mathrm{C}-\mathrm{V}$ profiles of samples after the annealing at $500{ }^{\circ} \mathrm{C}$. Now, the InAs planes containing the dots are more evidenced and more homogeneous in sample $\mathrm{A}$ as shown in Fig. 1c. Similar results were observed in sample B (Fig. 1d), where the C-V profile shows the contribution of three peaks related to the SAQDs. The broad peak in the C$\mathrm{V}$ profile of sample B was separated in two different peaks located at $166 \mathrm{~nm}$ and $168 \mathrm{~nm}$. After the annealing at $600{ }^{\circ} \mathrm{C}$, the $\mathrm{C}-\mathrm{V}$ profiles of samples $\mathrm{A}$ and $\mathrm{B}$ did not present any peculiarities related to the SAQDs, as depicted by the dot- 
ted lines in Figs. 1c and 1d. This is a first indication that the dots disappeared after the second annealing process $\left(600{ }^{\circ} \mathrm{C}\right)$ in these multilayer samples.

Due to the small separation between the InAs layers, the dots located in adjacent planes were vertically correlated during the growth ${ }^{9}$ and electronically coupled, which is

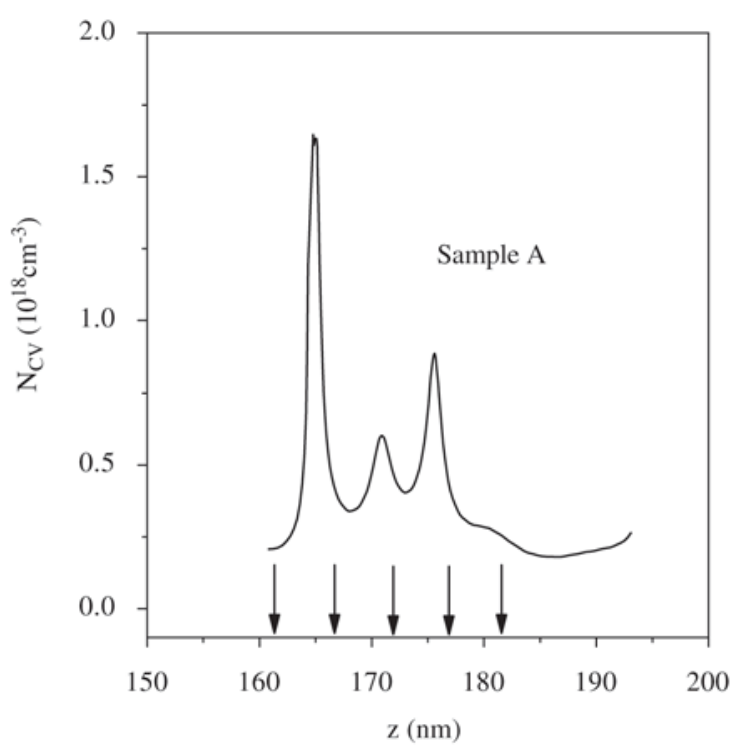

(a)

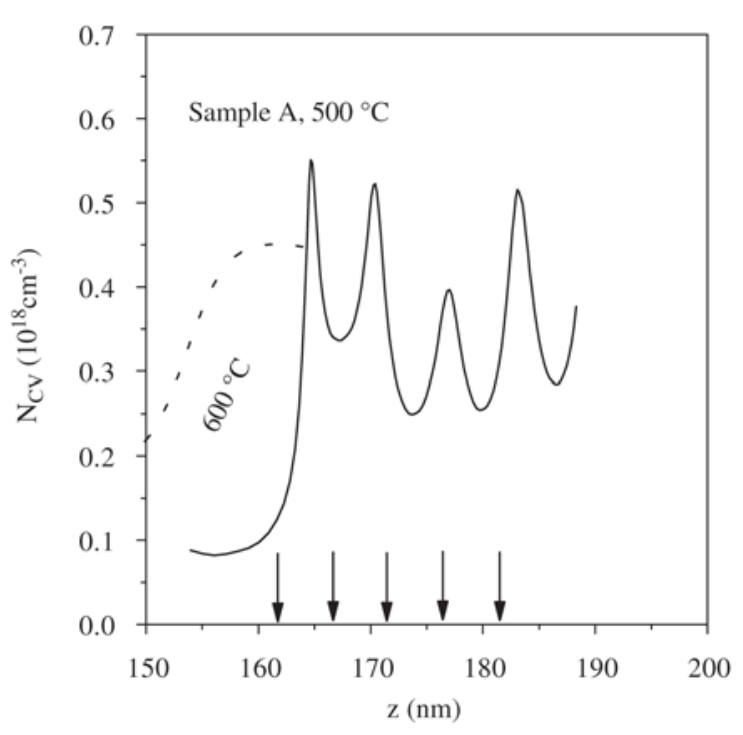

(c) evidenced by the nonvanishing electron density found in the barrier regions. Therefore, the applied electric field, liberating electrons in the InAs layers (starting at the surface of the sample) empties the other layers as well, thus masking their contribution to the measured capacitance. This effect certainly depends on the distances between the layers

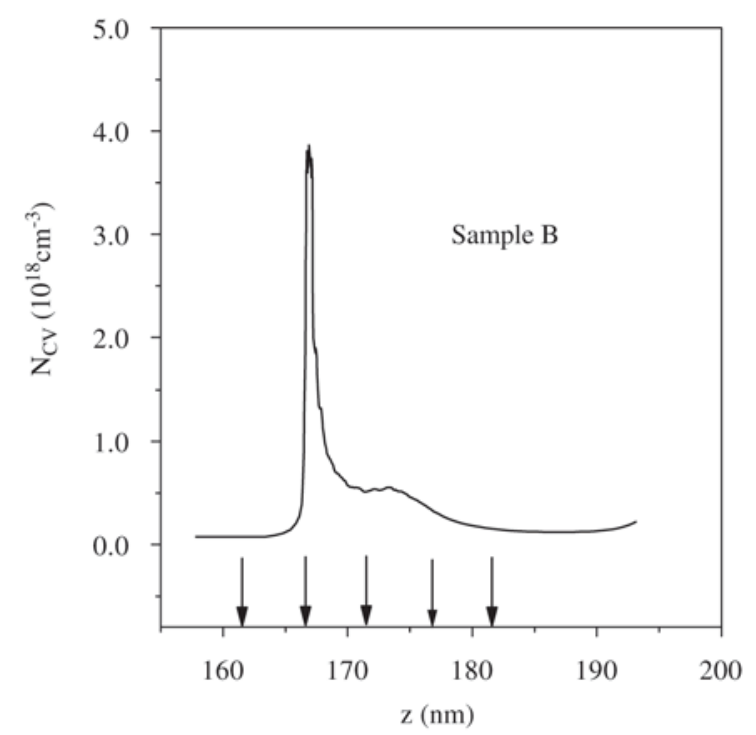

(b)

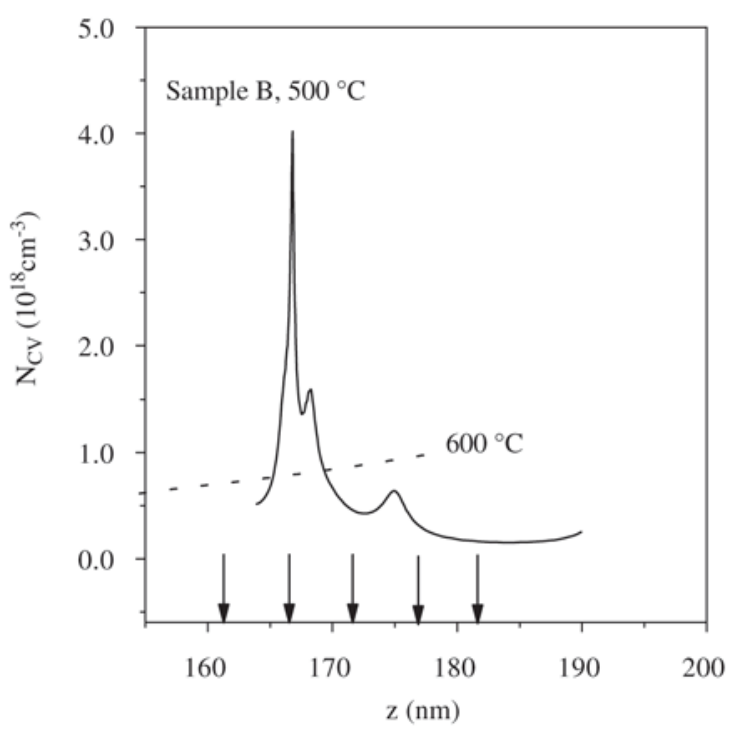

(d)

Figure 1. C-V profiles of the as-grown multilayer samples A and B are showed in panels a) and b). Panels c) and d) show the corresponding $\mathrm{C}-\mathrm{V}$ profiles after the annealing processes. Note that in the sample A (panel c)) the peaks have comparable intensities after the annealing. The dotted lines in panels c) and d) correspond to the annealing at $600^{\circ} \mathrm{C}$. Panel e) shows the $\mathrm{C}-\mathrm{V}$ profiles of the control sample at different annealing temperatures and panel $\mathrm{f}$ ) shows the $\mathrm{C}-\mathrm{V}$ profiles calculated for different doping levels in the GaAs barriers: $\mathrm{N}_{\mathrm{D}}=10^{20} \mathrm{~cm}^{-3}$ (full line) and $\mathrm{N}_{\mathrm{D}}=10^{19} \mathrm{~cm}^{-3}$ (dashed line). (Continues on next page) 


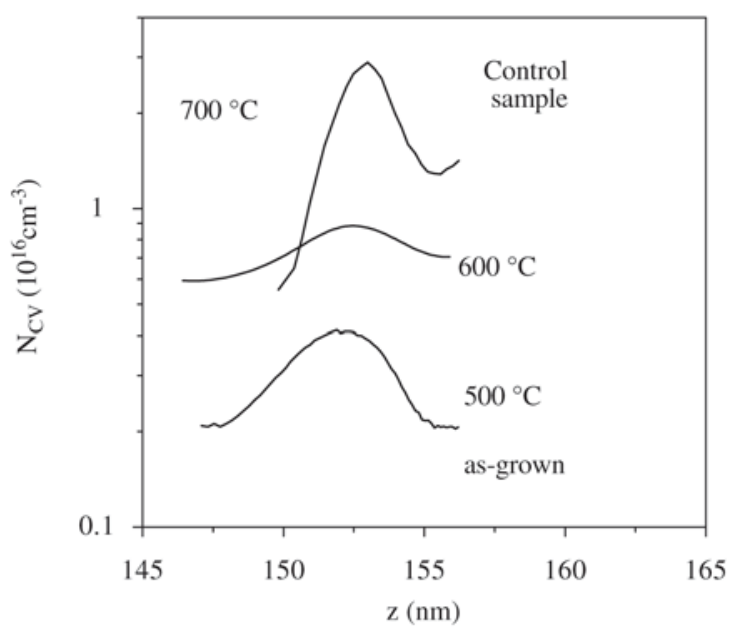

(e)

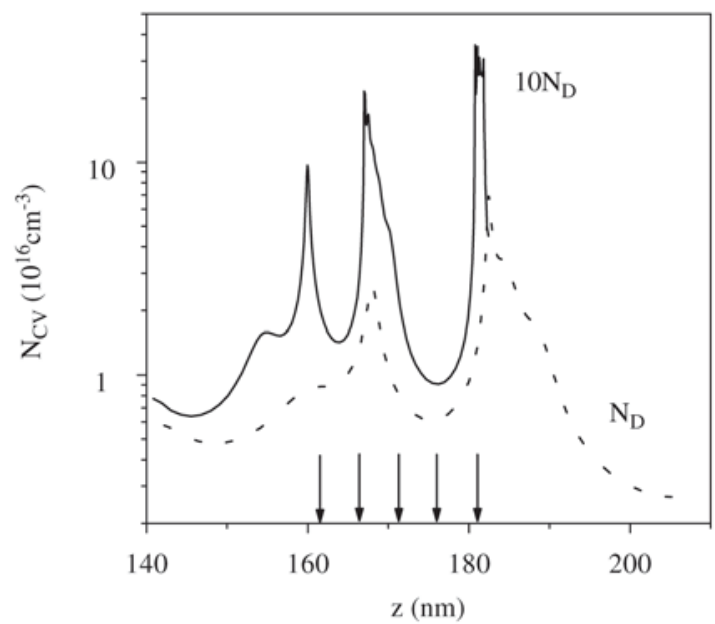

(f)

Figure 1. C-V profiles of the as-grown multilayer samples A and B are showed in panels a) and b). Panels c) and d) show the corresponding $\mathrm{C}-\mathrm{V}$ profiles after the annealing processes. Note that in the sample A (panel c)) the peaks have comparable intensities after the annealing. The dotted lines in panels c) and d) correspond to the annealing at $600{ }^{\circ} \mathrm{C}$. Panel e) shows the $\mathrm{C}-\mathrm{V}$ profiles of the control sample at different annealing temperatures and panel $\mathrm{f}$ ) shows the $\mathrm{C}-\mathrm{V}$ profiles calculated for different doping levels in the GaAs barriers: $\mathrm{N}_{\mathrm{D}}=10^{20} \mathrm{~cm}^{-3}$ (full line) and $\mathrm{N}_{\mathrm{D}}=10^{19} \mathrm{~cm}^{-3}$ (dashed line).

and on the dopant concentration ${ }^{10}$ : with the increase of the doping level the InAs dots planes become more electrically isolated thus showing the effect of screening (Figs. 1a,1b).

The corresponding PL spectra of the samples are depicted in Fig. 2a. They show no significant influence of the low temperature annealing at $500{ }^{\circ} \mathrm{C}$ (full lines) in comparison with the as-grown results (dotted lines). Although the low temperature annealing did not yet influence the properties of the whole multilayer SAQDs system (measured by PL), it significantly modified the individual properties of the layers (measured by $\mathrm{C}-\mathrm{V}$ ). This was clearly indicated by the increase of the electron localization inside the planes containing the dots $(\mathrm{C}-\mathrm{V}$ profiles observed after the annealing of $\left.500^{\circ} \mathrm{C}\right)$. The samples annealed at a higher temperature $\left(600^{\circ} \mathrm{C}\right)$ revealed a strong modification of the PL spectra: the intensities of the lines associated with the SAQDs drastically decreased and a new blue shifted broad line appeared around $1.3 \mathrm{eV}$.

The homogenization of the SAQDs and the increase in the localization of electrons observed by the $\mathrm{C}-\mathrm{V}$ measurements suggest possible structural alterations of the dots ${ }^{2-6}$. Using Raman scattering, sensitive to local structural modifications, we were able to confirm the reorganization of the SAQDs systems. The Raman spectra of samples with different doping levels, measured before and after the annealing process in the frequency range of the InAs optical phonons are shown in Fig. 3a. In both samples the TO mode was detected. The phonon lines are shifted due to the strain. In the configuration of back-scattering from the (001) surface we expect the singlet LO mode and the doublet TO mode to appear in the Raman spectra. The analysis of the strain field in the SAQDs ${ }^{11,12}$ shows that both the modes should present a blue shift. In Ref. 13 we have already found the TO and LO phonons in InAs/GaAs SAQDs at $250 \mathrm{~cm}^{-1}$ and $260 \mathrm{~cm}^{-1}$, respectively. Therefore, we here assign the line observed at $250 \mathrm{~cm}^{-1}$ to the TO phonon of InAs, while no contribution was found at the frequencies where the LO phonons of InAs were expected. The weak line found at $212 \mathrm{~cm}^{-1}$ is probably due to the disorder activated LA (DALA) phonon of GaAs. We did not find any significant shift of the bulk-like InAs phonon frequencies after the low temperature annealing process, which evidenced that the bulk structure of the SAQDs was not yet changed.

The Raman spectra measured in the frequency range of the GaAs optical phonons are presented in Fig. 3b. In this case both the LO and TO bulk phonons were clearly detected. In the as-grown samples they reveal red shifted frequencies due to the strain in the GaAs barriers.

In addition, a strong contribution of the GaAs-like optical phonons with large wave vectors reflecting the onephonon density of states was detected between the TO and LO lines. This defect induced Raman scattering decreased drastically in the samples annealed at $\mathrm{T}=600^{\circ} \mathrm{C}$ (Fig. 3b). It seems that the multilayer SAQDs structures caused a strong 


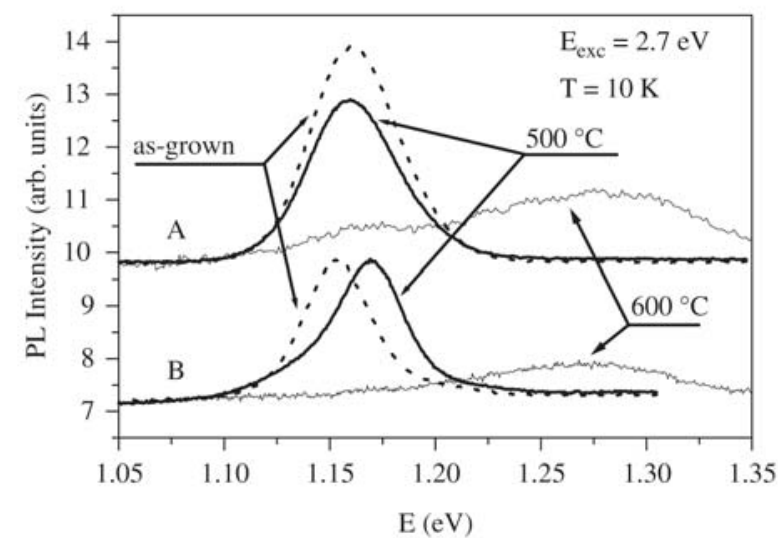

(a)

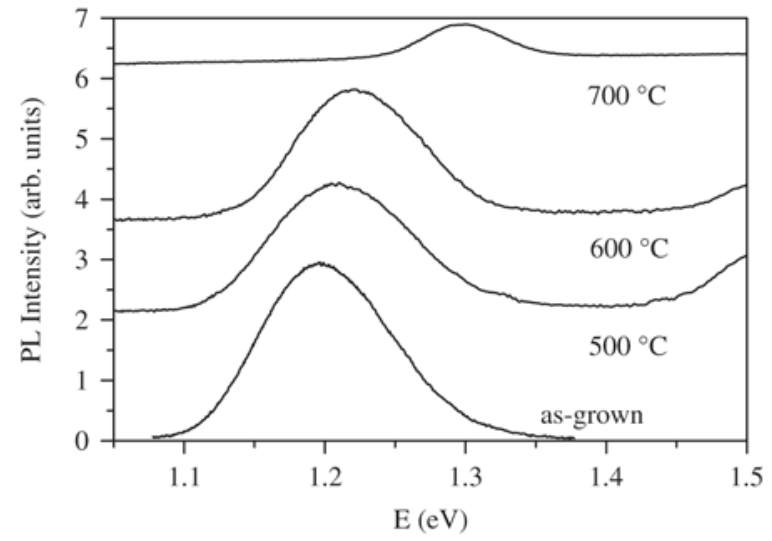

(b)

Figure 2. Panel a) shows the PL spectra of the as-grown multilayer samples A and B (dotted lines) taken at $10 \mathrm{~K}$. The corresponding spectra after the annealing at $500{ }^{\circ} \mathrm{C}$ are shown by full lines. The thin lines were obtained after the annealing at $600{ }^{\circ} \mathrm{C}$. Panel b) shows the PL spectra of the control sample with a single plane containing dots after the annealing at different temperatures. The curves were shifted up for clearness.

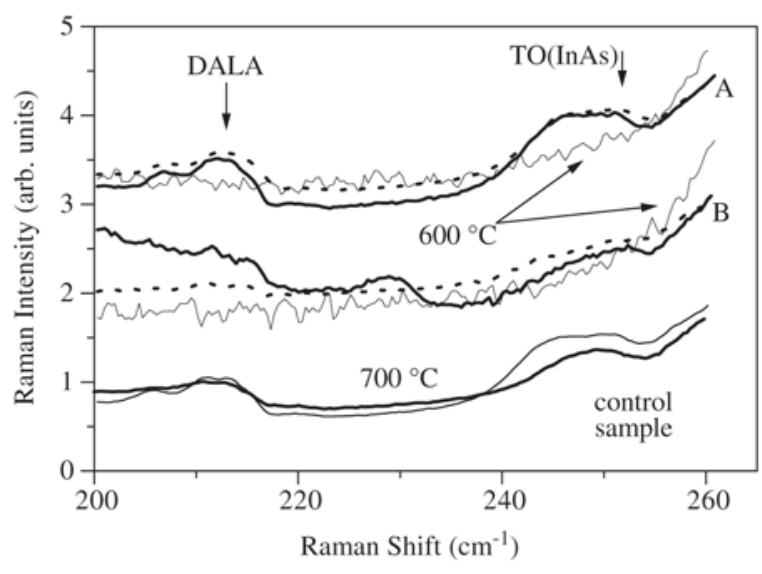

(a)

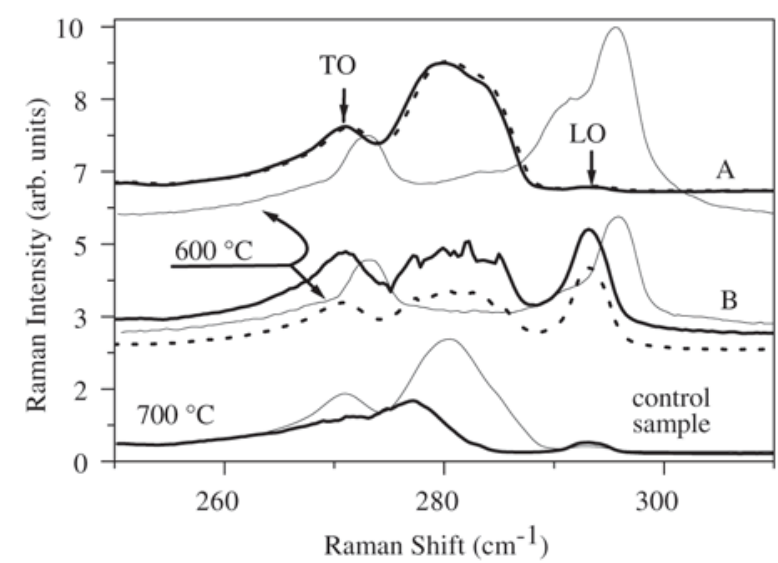

(b)

Figure 3. Raman spectra of the both multilayer and control samples. Panel a) shows the Raman spectra measured before and after the annealing process in the frequency range of the InAs optical phonons. The Raman spectra measured in the frequency range of the GaAs optical phonons are presented in panel (b). Full and dotted lines represent the annealed at $500{ }^{\circ} \mathrm{C}$ and as-grown samples, respectively. The thin lines were obtained after the annealing at $600{ }^{\circ} \mathrm{C}$. At the bottom of the figures the Raman spectra of both the as-grown and annealed $\left(700^{\circ} \mathrm{C}\right)$ control sample are presented.

violation of the Raman selection rules, while after the higher temperature annealing relatively homogeneous alloy layers were formed. Mostly the zone-center phonons of GaAs contributed to Raman scattering of the studied structures.

The observed modifications of the Raman spectra are explained as following: in the as-grown samples the defect induced Raman scattering of the optical phonons with large wave vectors dominate in the GaAs barriers, while the lines due to the bulk-like GaAs phonons (both TO and LO) reveal red shifted frequencies due to the strain. After the annealing at $600{ }^{\circ} \mathrm{C}$ the SAQDs disappeared and instead, homogeneous $\mathrm{In}_{\mathrm{x}} \mathrm{Ga}_{1-\mathrm{x}}$ As alloy layers were formed. As a result, the defect induced GaAs-like modes (DALA and the modes with large wave vectors) were eliminated. The shoulders found at the low frequency sides of the LO Raman lines correspond to the GaAs-like phonons of the 
$\mathrm{In}_{0.08} \mathrm{Ga}_{0.92}$ As alloy. We did not detect any evidences of the InAs-like phonons in the alloy.

As it was mentioned above, the PL spectra of the structures annealed at $600{ }^{\circ} \mathrm{C}$ show a strong blue shift and a broadening of the PL lines with significant decrease of their intensities (Fig. 2a). These shifted PL lines can be attributed to an $\operatorname{In}_{x} \mathrm{Ga}_{1-x}$ As alloy with $x \approx 0.10$ formed as a result of the annealing. This amount of In of the alloy agrees with the value found by Raman scattering. It is worth mentioning that the formation of the plasmon-LO phonon coupled modes is expected in the doped InAs/GaAs SAQDs structures subjected to the high temperature annealing due to the elimination of the localization potential of the SAQDs. This was not observed in our experiments probably due to the strong localization of the electrons in the InGaAs alloy now caused by defects.

Thus, we can conclude that the annealing at a temperature close to the growth temperature slightly improved the localization properties of the InAs/GaAs SAQDs system probably due to the increase of the concentration of free electrons (resulting in the decrease of the screening length) caused by the annealing of the point defects located in the GaAs barriers.

The annealing at $600{ }^{\circ} \mathrm{C}$ induced a diffusion process eliminating the SAQDs and forming an $\operatorname{In}_{x} \mathrm{Ga}_{1-\mathrm{x}}$ As alloy layer. A similar low-temperature diffusion process inducing a post-growth structural modification of the SAQDs was also detected in Ref. 5. We suppose that such low temperature diffusion was stimulated by the interlayer strain. In order to confirm this supposition we studied the effect of the annealing process in the single-layer SAQDs system (the control sample) and the results are depicted in Fig. 1e.

Additionally, we performed a simple qualitative simulation of the $\mathrm{C}-\mathrm{V}$ characteristics of the multilayered structures using the Poisson's equation with the electron density described by the Boltzmann's statistics. The results indeed show that an increase in the electron concentration in the barriers causes a reasonable improvement of the calculated $\mathrm{C}-\mathrm{V}$ profile (Fig. 1f) as it was obtained from the experimental measurements.

Figure $2 \mathrm{~b}$ shows the PL spectra of this sample after annealings at different temperatures. Contrary to the results obtained in the multilayer samples, even after the annealings at $600{ }^{\circ} \mathrm{C}$ and $700{ }^{\circ} \mathrm{C}$ we still observed the PL lines due the SAQDs, which were slightly blue shifted and broadened with the increase of the annealing temperature. Similar modifications of the PL lines were found in the annealed SAQDs in Refs. 2 - 4,6. The C-V data also revealed a thermal stability of the single-layer SADQs sample. Following our previous work ${ }^{14}$, the dispersion of the electron energy in the SAQDs $(\Delta \varepsilon)$ were calculated from the $\mathrm{C}-\mathrm{V}$ measurements. Both the calculated dispersion energies and the spectral widths of the PL lines decreased as the annealing tem- perature increased.

The Raman spectra of the single layer SAQDs sample shown in Figs. $3 \mathrm{a}$ and $3 \mathrm{~b}$ revealed the presences of the InAs optical phonons and the GaAs-like defect induced modes associated with the dots even after the annealing at $700{ }^{\circ} \mathrm{C}$. Thus, the low temperature annealing (at the temperature somewhat higher than the growth temperature) results in a drastic modification of the multilayer SAQDs, while the single layer structure revealed a thermal stability even at rather high temperatures (until $700^{\circ} \mathrm{C}$ ). These data confirm the role of the interlayer strain in the low temperature diffusion process.

\section{Conclusion}

In summary, we believe that the results presented in this paper constitute direct evidence that a post-growth annealing process can change the electronic properties of the multilayer SAQDs system. After a low temperature annealing, the individual contributions of each plane containing the dots were clearly seen. This implies in a decrease of the carrier coupling between the dots caused by the corresponding increase of the strength of the electron localization. In addition, we observed that after the annealing at $600{ }^{\circ} \mathrm{C}$ the InAs dots are diluted and the multilayer system produced an $\operatorname{In}_{x} \mathrm{Ga}_{1-\mathrm{x}}$ As alloy layer $(x \approx 0.10)$, while the single dot layer system revealed the presence of quantum dots even after the annealing at $600{ }^{\circ} \mathrm{C}$.

The authors would like to acknowledge E. Diagonel for the technical assistance. This work was partially supported by the Brazilian agencies, CNPq and FAPESP.

\section{References}

1. Goldstein,L.; Glas, F.; Marzin, J.Y.; Charasse, M.N.; LeRoux, G. Appl. Phys. Lett. 47, 1099, 1985.

2. Leon, R.; Kin, Y.; Jagadish, C.; Gal, M.; Zou, J. Cockayne, D.H. J. Appl. Phys. Lett. 69, 1888, 1996.

3. Ko sogov, A.O.; Werner, P.; Gösele, U.; Bimberg, N.; Ustinov, V.M.; Egorov, A.Y.; Zhuokov, A.E.; Kop'ev, P.S.; Bert, N.A.; Alferov, Z.I. Appl. Phys. Lett. 69, 3072, 1996.

4. Malik, S.; Roberts, C.; Murray, R. Pate, M. Appl. Phys. Lett. 71, 1987, 1997.

5. Johansson, J.; Seifert, W.; Zwiller, V.; Junno, T. Samuelson, L. Appl. Surf. Sci. v. 134, p. 47, 1998.

6. Fafard, S.; Wasilewski, Z.R.; Allen, C.Ni.; Picard, D.; Spaner, M.; McCaffrey, J.P.; Piva, P.G. Phys. Rev. B 59,15368, 1999.

7. Rhoderick, E.H.; Williams, R.H. Metal-semiconductor Contacts (Oxford, Cambridge) 1988.

8. Grundmann, M.; Christen, J.; Ledentsov, N.N.; Böhrer, J.; Bimberg, D.; Ruvimov, S.S.; Werner, P.; Richter, U.; Gösele, U.; Heydenreich, J.; Ustinov, V.M.; Egorov, A.Yu.; Zhukov, A.E.; Kopev, P.S.; Alferov, Zh.I. Phys. 
Rev. Lett. 74, 4043, 1995.

9. Xie, Q.; Madhukar, A.; Chen, P.; Kobayashi, N.P. Phys. Rev. Lett. 75, 2542, 1995.

10. Chiquito, A.J.; Pusep, Yu.A.; Mergulhão, S.; Galzerani, J.C.; Moshegov, N.T. Phys. Rev. B 61, 4481, 2000.

11. Cusak, M.A.; Briddon, P.R.; Jaros, M. Phys. Rev. B 54, R2300, 1996.
12. Grundmann, M.; Stier, O.; Bimberg, D. Phys. Rev. B 52, 11969, 1995.

13. Pusep, Yu.A.; Zanelatto, G.; Silva, S.W.; Galzerani, J.C.; Gonzalez-Borrero, P.P.; Toropov, A.I.; Basmaji, P. Phys. Rev. B 58, R1770, 1998.

14. Chiquito, A.J.; Pusep, Yu.A.; Mergulhão, S.; Galzerani, J.C.; Moshegov, N.T. Phys. Rev. B 61, 5499, 2000. 